\title{
Uncertain Random Simulation Algorithm with Application to Bottleneck Assignment Problem
}

DOI:

10.1007/s00500-018-3652-5

Document Version

Accepted author manuscript

Link to publication record in Manchester Research Explorer

\section{Citation for published version (APA):}

Ding, S., Zeng, X., \& Zhang, H. (2018). Uncertain Random Simulation Algorithm with Application to Bottleneck Assignment Problem. Soft Computing. https://doi.org/10.1007/s00500-018-3652-5

\section{Published in:}

Soft Computing

\section{Citing this paper}

Please note that where the full-text provided on Manchester Research Explorer is the Author Accepted Manuscript or Proof version this may differ from the final Published version. If citing, it is advised that you check and use the publisher's definitive version.

\section{General rights}

Copyright and moral rights for the publications made accessible in the Research Explorer are retained by the authors and/or other copyright owners and it is a condition of accessing publications that users recognise and abide by the legal requirements associated with these rights.

\section{Takedown policy}

If you believe that this document breaches copyright please refer to the University of Manchester's Takedown Procedures [http://man.ac.uk/04Y6Bo] or contact uml.scholarlycommunications@manchester.ac.uk providing relevant details, so we can investigate your claim.

\section{OPEN ACCESS}




\title{
Uncertain Random Simulation Algorithm with Application to Bottleneck Assignment Problem
}

\author{
Sibo Ding ${ }^{a, *}$ Xiao-Jun Zeng ${ }^{b}$ Huimin Zhang $^{a}$ \\ ${ }^{a}$ School of Management, Henan University of Technology, Zhengzhou 450001, China \\ ${ }^{b}$ School of Computer Science, University of Manchester, Manchester M13 9PL, UK
}

\begin{abstract}
Uncertain random simulation plays an important role in solving uncertain random optimization problems that include random variables and uncertain variables. In this paper, an uncertain random simulation is proposed and developed to obtain the chance distribution, $\alpha$-pessimistic value and $\alpha$-optimistic value. Further, an $\alpha$-optimal model for the uncertain random bottleneck assignment problem under the Hurwicz criterion is presented. Finally, a numerical example is given to illustrate how to use the proposed simulation algorithm to solve an uncertain random bottleneck assignment problem.
\end{abstract}

Keywords:

$\alpha$-pessimistic value; $\alpha$-optimistic value; uncertain random variable; assignment problem

\section{Introduction}

Uncertain random variable is introduced by Liu for modeling complex systems in which uncertain variables and random variables coexist [1]. For instance, when we have historical product sales data, we can estimate the probability distribution of the daily demand for this product. But when we sell a new product at the same time, we can not obtain the probability distribution of the daily demand for this new product due to lack of data. However, we can invite experts to estimate the daily demand for this new product according to their knowledge. In such a case, randomness and human uncertainty simultaneously coexist. To address such as a case, some scholars firstly studied the problem where there exists only human uncertainty. 
Liu founded uncertainty theory in 2007 [2]. Yang and Gao discuss uncertain differential game $[3,4]$. Gao and Qin calculate the edge-connectivity of an uncertain graph [5]. Dalman studies an uncertain multi-item solid transportation problem [6]. Hosseini and Wadbro carry out connectivity reliability analysis in uncertain networks [7]. In order to deal with uncertain random phenomenon, Liu proposes the chance theory [1]. Liu applies the chance theory to network optimization problem [8]. Dalman presents an uncertain random programming model for the fixed charge multi-item solid transportation problem [9]. Zhou et al. study the uncertain the random multi-objective programming [10]. Based on Taylor series, Dalman and Bayram reduce a class of multiobjective nonlinear programming problems into a single objective linear programming problem [11]. Ahmadzade et al. derive some properties of uncertain random partial quadratic entropy [12].

By using concepts and theorems of chance theory, Ke et al. investigate an uncertain random project scheduling problem [13]. They design an uncertain random simulation which randomly generates the sample points. However, their algorithm produces different values at different time. Sheng and Gao develop a simulation algorithm to solve uncertain random shortest path problem [14]. Their algorithm is designed specifically for uncertain random networks.

In this paper, we develop a new algorithm. Our algorithm uses the inverse uncertainty distribution and uniformly generates the sample points. It has better performances on the reliability and stability than the algorithm in [13]. In addition, our algorithm is a more general one than the previous algorithm in [14]. It can simulate the chance distribution function that is not only increasing with respect to some uncertain variables but also decreasing with respect to other uncertain variables.

The classic bottleneck assignment problem, which is raised from the parallel manufacturing systems, is how to assign the jobs to the machines such that the longest completion time is minimized. The classic bottleneck assignment problem is a deterministic one in which the completion times are constants. The deterministic bottleneck assignment problem is first introduced by Fulkerson et al. [15]. Gross proves the minmax theorem for the bottleneck assignment problem [16]. Researchers propose many solution methods for the bottleneck assignment problem. Threshold methods are proposed by to Edmonds and Fulkerson [17] and Garfinkel [18]. Carpaneto and Toth [19] develop a dual method. Other researchers study different bottleneck assignment problems. Malhotra et al. study three dimensional bottleneck 
assignment problem [20]. Aneja and Punnen consider a multiple bottleneck assignment problem [21]. Dokka et al. solve a multi-level bottleneck assignment problem [22].

Some other scholars investigate the random bottleneck assignment problem in which the costs are not constants but random variables. Yechiali is the first to investigate the random bottleneck assignment problem whose costs are independent random variables with exponential distributions [23]. Prabuddha et al. consider how to maximize the probability that the bottleneck time satisfies a specified threshold [24]. Pferschy studies the asymptotic behavior of the random bottleneck assignment problem [25]. Krokhmal and Pardalos provide a good overview of random bottleneck assignment problems [26]. Haus studies a random multidimensional bottleneck assignment problem and present a swapping algorithm [27].

In this paper, we propose a new uncertain random bottleneck assignment problem. It includes both random variables and uncertain variables. Our problem is different from the previous considered problems in [13-25]. In order to solve this problem, we combine uncertain random simulation with threshold techniques to design an algorithm.

The rest of this paper is organized as follows. In Section II, we introduce some basic knowledge on uncertainty theory and chance theory. In section III, we prove some formulas for uncertain random variables and present an uncertain random simulation algorithm. In Section IV, we propose an uncertain random bottleneck assignment model under the Hurwicz criterion. Then, an algorithm to solve this problem is presented. At the end of the paper, a conclusion section is given.

\section{Preliminaries}

\subsection{Uncertainty Theory}

Definition 1. (Liu [28]): An uncertainty distribution $\Phi(x)$ is said to be regular if it is a continuous and strictly increasing function with respect to $x$ at which $0<\Phi(x)<1$, and

$$
\lim _{x \rightarrow-\infty} \Phi(x)=0, \lim _{x \rightarrow+\infty} \Phi(x)=1 .
$$

Definition 2. (Liu [28]): Let $\xi$ be an uncertain variable with regular uncertainty distribution $\Phi(x)$. Then the inverse function $\Phi^{-1}(\alpha)$ is called the inverse uncertainty distribution of $\xi$. 
Theorem 1. (Liu [28]): Let $\xi_{1}, \xi_{2}, \cdots, \xi_{n}$ be independent uncertain variables with regular uncertainty distributions $\Phi_{1}, \Phi_{2}, \ldots, \Phi_{n}$, respectively. If $f\left(\xi_{1}, \xi_{2}, \cdots, \xi_{n}\right)$ is strictly increasing with respect to $\xi_{1}, \xi_{2}, \cdots, \xi_{m}$ and strictly decreasing with respect to $\xi_{m+1}, \xi_{m+2}, \cdots, \xi_{n}$, then

$$
\xi=f\left(\xi_{1}, \xi_{2}, \cdots, \xi_{n}\right)
$$

has an inverse uncertainty distribution.

$$
\begin{aligned}
\Psi^{-1}(\alpha)= & f\left(\Phi_{1}^{-1}(\alpha), \cdots, \Phi_{m}^{-1}(\alpha),\right. \\
& \left.\Phi_{m+1}^{-1}(1-\alpha), \cdots, \Phi_{n}^{-1}(1-\alpha)\right) .
\end{aligned}
$$

\subsection{Chance Theory}

Definition 3. (Liu [1]): Let $(\Gamma, \mathcal{L}, \mathcal{M})$ be an uncertainty space and let $(\Omega, \mathcal{A}, \operatorname{Pr})$ be a probability space. Then the product $(\Gamma, \mathcal{L}, \mathcal{M}) \times(\Omega, \mathcal{A}, \operatorname{Pr})$ is called a chance space

$$
(\Gamma, \mathcal{L}, \mathcal{M}) \times(\Omega, \mathcal{A}, \operatorname{Pr})=(\Gamma \times \Omega, \mathcal{L} \times \mathcal{A}, \mathcal{M} \times \operatorname{Pr}) .
$$

Theorem 2. (Liu [29]): Let $\eta_{1}, \eta_{2}, \cdots, \eta_{m}$ be independent random variables with probability distributions $\Psi_{1}, \Psi_{2}, \cdots, \Psi_{m}$, respectively, and let $\tau_{1}, \tau_{2}, \cdots, \tau_{n}$ be independent uncertain variables. Assume $f$ is a measurable function. Then the uncertain random variable

$$
\xi=f\left(\eta_{1}, \eta_{2}, \cdots, \eta_{m}, \tau_{1}, \tau_{2}, \cdots, \tau_{n}\right)
$$

has a chance distribution

$$
\begin{gathered}
\Phi(x)=\int_{\Re^{m}} F\left(x ; y_{1}, y_{2}, \cdots, y_{m}\right) \mathrm{d} \Psi_{1}\left(y_{1}\right) \\
\mathrm{d} \Psi_{2}\left(y_{2}\right) \cdots \mathrm{d} \Psi_{m}\left(y_{m}\right) .
\end{gathered}
$$

where $F\left(x ; y_{1}, y_{2}, \cdots, y_{m}\right)$ is the uncertainty distribution of the variable

$$
f\left(y_{1}, y_{2}, \cdots, y_{m}, \tau_{1}, \tau_{2}, \cdots, \tau_{n}\right) .
$$

Definition 4. (Liu [1]): Let $\xi$ be an uncertain random variable. Then its chance distribution is defined by

$$
\Phi(x)=\operatorname{Ch}\{\xi \leq x\}
$$

for any $x \in \Re$.

In order to obtain the chance distribution, we prove following theorems and develop a simulation algorithm. 


\section{Uncertain random simulation}

In fact, Formula (3) is a theoretical one, which is not easy to use in most cases due to the complexity of chance distribution function. To overcome the difficulty, an uncertain random simulation is proposed to construct the chance distribution. First, we introduce the concepts of $\alpha$-pessimistic value and $\alpha$-optimistic value for an uncertain random variable. Then, we obtain the chance distribution, $\alpha$-pessimistic value and $\alpha$-optimistic value by using a numerical integration method.

Definition 5. Let $\xi$ be an uncertain random variable on chance space $(\Gamma, \mathcal{L}, \mathcal{M}) \times$ $(\Omega, \mathcal{A}, \operatorname{Pr})$ and $\alpha \in(0,1]$. Then,

$$
\xi_{\text {inf }}(\alpha)=\inf \{r \mid \operatorname{Ch}\{\xi \leq r\} \geq \alpha\}
$$

and

$$
\xi_{\text {sup }}(\alpha)=\sup \{r \mid \operatorname{Ch}\{\xi \geq r\} \geq \alpha\}
$$

are called the $\alpha$-pessimistic value and the $\alpha$-optimistic value of $\xi$, respectively.

Theorem 3. Let $\xi$ be an uncertain random variable and $\alpha \in(0,1]$. Then, we have

$$
\xi_{\text {inf }}(\alpha)=\Phi^{-1}(\alpha)
$$

Proof: It follows from Definition 5 immediately.

Theorem 4. Let $\xi$ be an uncertain random variable and $\alpha \in(0,1]$. Then, we have

$$
\xi_{\text {inf }}(\alpha)=\xi_{\text {sup }}(1-\alpha) .
$$

Proof: It follows from Equation (6) that

$$
\operatorname{Ch}\left\{\xi \geq \xi_{\text {sup }}(1-\alpha)\right\}=1-\alpha .
$$

Thus,

$$
\begin{aligned}
\operatorname{Ch}\left\{\xi \leq \xi_{\text {sup }}(1-\alpha)\right\} & =1-\operatorname{Ch}\left\{\xi \geq \xi_{\text {sup }}(1-\alpha)\right\} \\
& =1-(1-\alpha) \\
& =\alpha
\end{aligned}
$$

Thus, we have $\xi_{\text {inf }}(\alpha)=\xi_{\text {sup }}(1-\alpha)$. The theorem is proved. 
Theorem 5. Let $\xi$ be an uncertain random variable and $\alpha \in(0,1]$. Then, we have

$$
\xi_{\text {sup }}(\alpha)=\Phi^{-1}(1-\alpha) \quad \text { and } \quad \xi_{\text {sup }}(1-\alpha)=\Phi^{-1}(\alpha) .
$$

Proof: It follows from Theorems 3 and 4 .

According to Theorems 3, 4 and 5, we design the following uniform discretization algorithm to calculate $\Phi(x), \alpha$-pessimistic value and $\alpha$-optimistic value. Our algorithm is very flexible. It can even create the empirical distribution.

\section{Algorithm 1 (Uniform Discretization Algorithm)}

Step 1. Discretize the range of the random variable $\eta_{i}$ into $N_{i}$ equally spaced points.

Step 2. Discretize $\alpha$ into $K$ equally spaced points.

Step 3. Calculate $F^{-1}\left(\alpha ; y_{1}, y_{2}, \cdots, y_{m}\right)$.

Step 4. Calculate $F\left(x ; y_{1}, y_{2}, \cdots, y_{m}\right)=$

$$
\left\{\begin{array}{lll}
0 & \text { if } \quad x \leq x_{1}, \\
\alpha_{i}+\left(\alpha_{i+1}-\alpha_{i}\right) \frac{x-x_{i}}{x_{i+1}-x_{i}} & \text { if } \quad & x_{i} \leq x \leq x_{i+1} \\
& & 1 \leq i \leq K, \\
1 & \text { if } \quad x \geq x_{K},
\end{array}\right.
$$

Step 5. Apply numerical integration to calculate $\Phi(x), \alpha$-pessimistic value and $\alpha$-optimistic value.

We illustrate the uniform discretization algorithm by the following example.

Example 1. Suppose that $\eta_{1}$ and $\eta_{2}$ are independent random variables with probability distributions $U(1,2)$ and $U(2,4)$, and suppose that $\tau_{1}$ and $\tau_{2}$ are independent uncertain variables with uncertainty distributions $\mathcal{L}(1,5)$ and $\mathcal{L}(1,3)$. Then $\xi=\eta_{1}+\eta_{2}+\tau_{1}-\tau_{2}$ is an uncertain random variable. Assume that $\xi$ has chance distribution $\Phi(x)$.

For the sake of simplicity, we set $N_{1}=10, N_{2}=10, K=10$. We also can assign a large number to $N_{1}, N_{2}$ and $K$ to improve accuracy. The probability distribution function of $\eta_{1}$ and $\eta_{2}$ are

$$
\Psi_{1}\left(y_{1}\right)= \begin{cases}0 & \text { if } y_{1} \leq 1 \\ y_{1}-1 & \text { if } 1 \leq y_{1} \leq 2, \text { and } \\ 1 & \text { if } \quad y_{1} \geq 2\end{cases}
$$




$$
\Psi_{2}\left(y_{2}\right)=\left\{\begin{array}{lll}
0 & \text { if } & y_{2} \leq 2 \\
\frac{y_{2}-2}{2} & \text { if } & 2 \leq y_{2} \leq 4 \\
1 & \text { if } & y_{2} \geq 4
\end{array}\right.
$$

Then, we can have discrete forms of $\Psi_{1}\left(y_{1}\right)$ and $\Psi_{1}\left(y_{2}\right)$ in which $y_{1}=$ $1+0.1 \cdot i$ and $y_{2}=2+0.2 \cdot j$ for $i=1,2, \cdots, 10$ and $j=1,2, \cdots, 10$. The inverse uncertainty distribution function of $\tau_{1}$ and $\tau_{2}$ are $\left(\tau_{1}\right)_{\text {inf }}(\alpha)=$ $(1-\alpha) \cdot 1+\alpha \cdot 5=1+4 \cdot \alpha$ and $\left(\tau_{2}\right)_{\inf }(1-\alpha)=\alpha \cdot 1+(1-\alpha) \cdot 3=3-2 \cdot \alpha$.

The chance distribution of $\xi$ is

$$
\Phi(x)=\frac{1}{2} \int_{2}^{4} \int_{1}^{2} F\left(x ; y_{1}, y_{2}\right) \mathrm{d} y_{1} \mathrm{~d} y_{2}
$$

where $F\left(x ; y_{1}, y_{2}\right)$ is obtained by the inverse uncertainty distribution function $F^{-1}\left(\alpha ; y_{1}, y_{2}\right)=y_{1}+y_{2}+(1+4 \cdot \alpha)-(3-2 \cdot \alpha)$ for each $\alpha \in(0,1]$. This implies that $F^{-1}\left(\alpha ; y_{1}, y_{2}\right)=x_{k}, 1 \leq k \leq 10$ for each $\alpha \in(0,1]$. The value of $F^{-1}\left(\alpha ; y_{1}, y_{2}\right)$ is listed on the Table I.

Table 1 Inverse uncertainty distribution function $F^{-1}\left(\alpha ; y_{1}, y_{2}\right)$

\begin{tabular}{rrllcl}
\hline$i$ & $j$ & $y_{1}=1+0.1 \cdot i$ & $y_{2}=2+0.2 \cdot j$ & $\alpha$ & $F^{-1}$ \\
\hline 1 & 1 & 1.1 & 2.2 & 0.1 & 1.9 \\
1 & 1 & 1.1 & 2.2 & 0.2 & 2.5 \\
$\ldots$ & $\ldots$ & $\ldots$ & $\ldots$ & $\ldots$ & $\ldots$ \\
1 & 1 & 1.1 & 2.2 & 1 & 7.3 \\
\hline 1 & 2 & 1.1 & 2.4 & 0.1 & 2.1 \\
1 & 2 & 1.1 & 2.4 & 0.2 & 2.7 \\
$\ldots$ & $\ldots$ & $\ldots$ & $\ldots$ & $\ldots$ & $\ldots$ \\
1 & 2 & 1.1 & 2.4 & 1 & 7.5 \\
$\ldots$ & $\ldots$ & $\ldots$ & $\ldots$ & $\cdots$ & $\cdots$ \\
10 & 10 & 2 & 4 & 0.1 & 4.6 \\
$\ldots$ & $\ldots$ & $\ldots$ & $\ldots$ & $\cdots$ & $\ldots$ \\
10 & 10 & 2 & 4 & 1 & 10 \\
\hline
\end{tabular}

Then, according to Step 4 , we obtain $F\left(x ; y_{1}, y_{2}\right)$. Now, we get the chance 
distribution function of $\xi$.

$$
\begin{aligned}
\Phi(x) & =\frac{1}{2} \int_{2}^{4} \int_{1}^{2} F\left(x ; y_{1}, y_{2}\right) \mathrm{d} y_{1} \mathrm{~d} y_{2} \\
& =\frac{1}{2} \sum_{i=1}^{10} \sum_{j=1}^{10} F(x ; 1+0.1 \cdot i, 2+0.2 \cdot j) \cdot 0.1 \cdot 0.2
\end{aligned}
$$

By Uniform Discretization Algorithm, we get the chance distribution of $\xi$ (Fig. 1), $\xi_{\text {inf }}(0.2)=3.9$ and $\xi_{\text {sup }}(0.2)=7.4$.

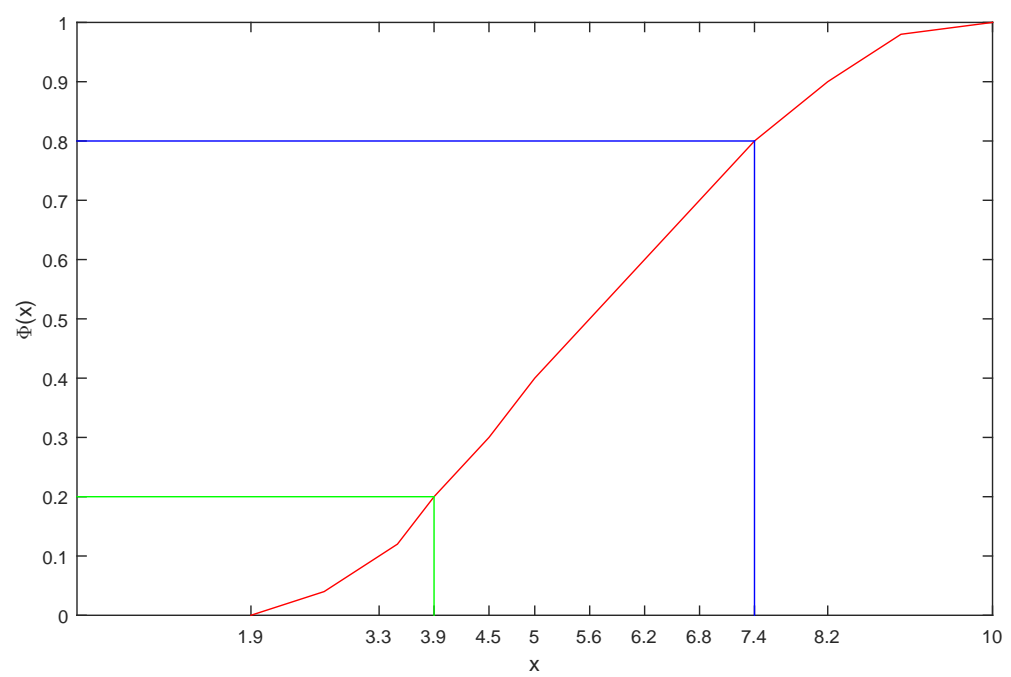

Fig. 1. The chance distribution of $\xi$.

The advantage of our simulation algorithm is that it is easy to implement and provides a way to calculate the $\alpha$-optimistic and $\alpha$-pessimistic values for all types of uncertain random variables. The disadvantage is that, as the number of variables increases, much more storage space is needed.

In the next section, we will use the proposed uncertain random simulation to solve the uncertain random bottleneck assignment problem. An $\alpha$-optimal model will be presented and an algorithm will be designed to obtain the solution for the $\alpha$-optimal model. 


\section{Uncertain random bottleneck assignment model}

The uncertain random bottleneck assignment problem is defined as follows:

$$
\begin{aligned}
\min \max _{1 \leq i, j \leq n} & \left(\rho\left(\xi_{i j}\right)_{\sup }(\alpha)+(1-\rho)\left(\xi_{i j}\right)_{\inf }(\alpha)\right) x_{i j} \\
\text { s.t. } & \operatorname{Ch}\left\{\xi_{i j} \geq\left(\xi_{i j}\right)_{\sup }(\alpha)\right\} \geq \alpha \\
& \operatorname{Ch}\left\{\xi_{i j} \leq\left(\xi_{i j}\right)_{\inf }(\alpha)\right\} \geq \alpha \\
& \sum_{j=1}^{n} x_{i j}=1, i=1,2,3, \ldots, n, \\
& \sum_{i=1}^{n} x_{i j}=1, j=1,2,3, \ldots, n, \\
& x_{i j}=0 \text { or } 1, i, j=1,2,3, \ldots, n
\end{aligned}
$$

where the cost coefficient $\xi_{i j}$ is the completion time of assigning men $j$ to task $i . x_{i j}$ is an indicator of whether or not man $j$ is assigned to do the task $i$.

We apply Hurwicz criterion to model the uncertain random bottleneck assignment problem. The Hurwicz criterion under uncertain random environment is $\rho \xi_{\text {sup }}(\alpha)+(1-\rho) \xi_{\text {inf }}(\alpha)$, where $\xi_{\text {sup }}(\alpha)$ and $\xi_{\text {inf }}(\alpha)$ are the $\alpha$-optimistic completion time and $\alpha$-pessimistic completion time. It means that the completion time is less than $\xi_{\text {sup }}(\alpha)$ and more than $\xi_{\text {inf }}(\alpha) . \alpha$ is the belief degree of accomplishing a task. Under the Hurwicz criterion, the decision payoffs are weighted by a coefficient of optimism $\rho$. When $\rho=1$, the criterion is the optimistic one. When $\rho=0$, it is a pessimistic criterion.

The essential idea of the $\alpha$-optimal model is to arrive at a rational compromise between the optimistic and pessimistic criterions subject to chance constraints. Chance constraint (12) represents that the chance that the $\alpha$ optimistic time is shorter than the completion time is greater than $\alpha$. Chance constraint (13) shows that the chance that the $\alpha$-pessimistic time is longer than completion time is greater than $\alpha$.

In summary, there are three main differences between our model and previous models. First, in order to describe the uncertain random phenomena, uncertain random variables are added into our model. Second, the objective function is formulated according to the Hurwicz criterion. Third, new chance constraints are contained in our model. 
Considering the above uncertain random bottleneck assignment model, we propose the following algorithm in which uncertain random simulation is used to simulate uncertain random cost coefficients.

\section{Algorithm 2 (Uncertain Random Bottleneck Assignment Algo- rithm)}

Step 1. Set values of $\alpha$ and $\rho$.

Step 2. Apply Uniform Discretization Algorithm to calculate $\left(\xi_{i j}\right)_{\text {sup }}(\alpha)$ and $\left(\xi_{i j}\right)_{\text {inf }}(\alpha)$. Compute $w_{i j}=\rho\left(\xi_{i j}\right)_{\text {sup }}(\alpha)+(1-\rho)\left(\xi_{i j}\right)_{\text {inf }}(\alpha)$ and let $\mathrm{W}=\left(w_{i j}\right)$.

Step 3. Set $\underline{a}=\min (\mathrm{W})$ and $\bar{a}=\max (\mathrm{W})$.

Step 4. If find the median $a^{*}$ between $\underline{a}$ and $\bar{a}$ in $\mathrm{W}$, goto Step5. Otherwise, goto Step 7.

Step 5. Let $w_{i j}$ with $w_{i j} \leq a^{*}$ be admissible and create a new threshold matrix C.

Step 6. If find a perfect matching in C by using Hungarian method, set $\bar{a}=a^{*}$, goto Step 4. Otherwise, set $\underline{a}=a^{*}$, goto Step 4 .

Step 7. Stop. The optimal value is $a^{*}$ and the optimal assignment is obtained.

We illustrate Algorithm 2 through the next numerical example.

Example 2. Suppose that $\eta_{1}, \eta_{2}, \cdots, \eta_{5}$ are independent random variables and $\tau_{1}, \tau_{2}, \cdots, \tau_{7}$ are independent uncertain variables. They are given as the following Table II.

Table 2 Random variables and uncertain variables in Example 2

\begin{tabular}{llll}
\hline$i$ & $\eta_{i}$ & $j$ & $\tau_{j}$ \\
\hline 1 & $\eta_{1} \sim U(1,2)$ & 1 & $\tau_{1} \sim \mathcal{L}(2,4)$ \\
2 & $\eta_{2} \sim U(3,4)$ & 2 & $\tau_{2} \sim 5$ \\
3 & $\eta_{3} \sim U(3,4)$ & 3 & $\tau_{3} \sim \mathcal{L}(2,3)$ \\
4 & $\eta_{4} \sim U(4,5)$ & 4 & $\tau_{4} \sim \mathcal{L}(5,8)$ \\
5 & $\eta_{5} \sim U(5,6)$ & 5 & $\tau_{5} \sim 7$ \\
- & - & 6 & $\tau_{6} \sim \mathcal{L}(9,10)$ \\
- & - & 7 & $\tau_{7} \sim \mathcal{L}(1,2)$ \\
\hline
\end{tabular}


Random variables and uncertain variables are special uncertain random variables. Uncertain random variables are listed in the following cost matrix

$$
\boldsymbol{\xi}=\left(\begin{array}{lll}
\tau_{6} & \eta_{1}+\tau_{1} & \tau_{2} \\
\eta_{1}+\tau_{1} & \tau_{5} & \eta_{3}+\tau_{3} \\
\eta_{2} & \eta_{4}+\eta_{5}+2 \cdot \tau_{7} & \tau_{4}
\end{array}\right)
$$

Let us explain the meaning of the matrix element. For instance, $\xi_{32}$ means man 2 needs $\eta_{4}+\eta_{5}+2 \cdot \tau_{7}$ time in order to do task 3 . The values of variables $\eta_{4}$ and $\eta_{5}$ are the outcomes of statistical experiments. Variables $\eta_{4}$ and $\eta_{5}$ are random variables. The value of variable $\tau_{7}$ is the outcome of expert estimation. Variable $\tau_{7}$ is an uncertain variable. Thus, $\xi_{32}$ is an uncertain random variable.

Here let us assume $\alpha=0.4$ and $\rho=0.1$. Of course, we can choose different values according to the requirements of a decision maker. Let $w_{i j}=$ $\rho\left(\xi_{i j}\right)_{\text {sup }}(\alpha)+(1-\rho)\left(\xi_{i j}\right)_{\text {inf }}(\alpha)$. Tabel III lists the $\alpha$-pessimistic value, the $\alpha$-optimistic value and cost $w_{i j}$.

Table $3 \quad \alpha$-pessimistic value, $\alpha$-optimistic value and cost $w_{i j}$

\begin{tabular}{ccccc}
\hline$i$ & $j$ & $\left(\xi_{i j}\right)_{\text {sup }}(\alpha)$ & $\left(\xi_{i j}\right)_{\text {inf }}(\alpha)$ & $w_{i j}$ \\
\hline 1 & 1 & 9.6 & 9.4 & 9.42 \\
1 & 2 & 5.34 & 4.35 & 4.45 \\
1 & 3 & 5 & 5 & 5 \\
2 & 1 & 5.34 & 4.35 & 4.45 \\
2 & 2 & 7 & 7 & 7 \\
2 & 3 & 6.15 & 5.95 & 5.97 \\
3 & 1 & 3.46 & 2.54 & 2.63 \\
3 & 2 & 13.3 & 12.9 & 12.94 \\
3 & 3 & 6.8 & 6.2 & 6.26 \\
\hline
\end{tabular}

Then, we can obtain the cost matrix

$$
\boldsymbol{W}=\left(\begin{array}{ccc}
9.42 & 4.45 & 5 \\
4.45 & 7 & 5.97 \\
2.63 & 12.94 & 6.26
\end{array}\right)
$$


We find $\underline{a}=2.63, \bar{a}=12.94$ and $a^{*}=5.97$.

Thus, the threshold matrix becomes

$$
\boldsymbol{C}=\left(\begin{array}{ccc}
\times & 4.45 & 5 \\
4.45 & \times & 5.97 \\
2.63 & \times & \times
\end{array}\right)
$$

The maximum matching has cardinality 3. Then, we repeat Step 4 to Step 6. Finally, we obtain that it is optimal to assign Man 1 to Task 3, Man 2 to Task 1 and Man 3 to Task 2. The final completion time associated with this solution is $a^{*}=5.97$. This completes the solution of the problem.

\section{Conclusion}

In this paper, an uncertain random simulation algorithm is designed to construct the chance distribution. Our uncertain random simulation can be used in the uncertain random optimization for solving chance constraints. Further, we propose an uncertain random bottleneck assignment model under the Hurwicz criterion, in which random variables and other uncertain variables coexist. We then apply the proposed uncertain random simulation algorithm to optimize the model. A numerical example is presented to illustrate the algorithm. The uncertain random simulation algorithm can be used to obtain the chance distributions for all kinds of uncertain random variables with high accuracy. However, when the number of uncertain random variables increases, a large size of storage spaces is needed. How to overcome such a limitation is one of our future research topics.

\section{Acknowledgments}

This work was supported by the National Natural Science Foundation of China (Grant no. U1404701), the Scholarship Program of China Scholarship Council (Grant no. 201509895007), the Scientific Research Foundation of the Henan University of Technology (Grant no. 2017RCJH11) and the Key Research Institute of Humanities and Social Sciences at Universities of Henan (Grant no. 2015-JD-04). We are grateful to anonymous reviewers for their thoughtful comments, which help considerably to improve the presentation of this work. 


\section{Compliance with ethical standards}

\section{Conflict of interest}

The authors declare that they have no conflict of interest.

\section{Human and animal rights}

This article does not contain any studies with human participants or animals performed by any of the authors.

\section{References}

[1] Y. H. Liu, Uncertain random variables: A mixture of uncertainty and randomness, Soft Comput. 17 (2013) 625-634.

[2] B. Liu, Uncertainty Theory, 2nd ed., Springer-Verlag, Berlin, Germany, 2007.

[3] X. F. Yang, J. W. Gao, Linear-quadratic uncertain differential game with application to resource extraction problem, IEEE T. Fuzzy Syst. 24 (2016) 819-826.

[4] J. W. Gao, Uncertain bimatrix game with applications, Fuzzy Optim. Decis. Ma. 12 (2013) 65-78.

[5] Y. Gao, Z. F. Qin, On computing the edge-connectivity of an uncertain graph, IEEE T. Fuzzy Syst. 24 (2016) 981-991.

[6] H. Dalman, Uncertain programming model for multi-item solid transportation problem, Int. J. Mach. Learn. Cyb. 9 (4) ( 2018) 559-567.

[7] S. A. Hosseini, E. Wadbro, Connectivity reliability in uncertain networks with stability analysis, Expert Syst. Appl. 57 (2016) 337-344.

[8] B. Liu, Uncertain random graph and uncertain random network, J. Uncertain Syst. 8 (2014) 3-12.

[9] H. Dalman, Uncertain random programming models for fixed charge multi-item solid transportation problem, New Trends Math. Sci. 6 (1) (2018) 37-51.

[10] J. Zhou, F. Yang, K. Wang, Multi-objective optimization in uncertain random environments, Fuzzy Optim. Decis. Ma. 13 (2014) 397-413. 
[11] H. Dalman, M. Bayram, Interactive fuzzy goal programming based on Taylor series to solve multiobjective nonlinear programming problems with interval type 2 fuzzy numbers, IEEE T. Fuzzy Syst. 26 (4) (2018) 24342449 .

[12] H. Ahmadzade, R. Gao, H. Zarei, Partial quadratic entropy of uncertain random variables, J. Uncertain Syst. 10 (2016) 292-301.

[13] H. Ke, H. Liu, G. G. Tian, An uncertain random programming model for project scheduling problem, Int. J. Intell. Syst. 30 (2015) 66-79.

[14] Y. H. Sheng, Y. Gao, Shortest path problem of uncertain random network, Comput. Ind. Eng. 99 (2016) 97-105.

[15] D. R. Fulkerson, I. Glicksberg, O. Gross, A production line assignment problem, Tech. Rep. RM-1102, The Rand Corporation, Santa Monica, CA, 1953.

[16] O. Gross, The bottleneck assignment problem, Tech. Rep. P-1630, The Rand Corporation, Santa Monica, CA, 1959.

[17] J. Edmonds, D. R. Fulkerson, Bottleneck extrema, J. Comb. Theory 8 (1970) 299-306.

[18] R. Garfinkel, An improved algorithm for the bottleneck assignment problem, Oper. Res. 19 (1971) 1747-1751.

[19] G. Carpaneto, P. Toth, Algorithm for the solution of the bottleneck assignment problem, Comput. 27 (1981) 179-187.

[20] R. Malhotra, H. L. Bhatia, M. C. Puri, The three dimensional bottleneck assignment problem and its variants, Optimization 16 (1985) 245-256.

[21] Y. Aneja, A. Punnen, Multiple bottleneck assignment problem, Eur. J. Oper. Res. 112 (1999) 167-173.

[22] T. Dokka, A. Kouvela, F. Spieksma, Approximating the multi-level bottleneck assignment problem, Oper. Res. Lett. 40 (2012) 282-286.

[23] U. Yechiali, A stochastic bottleneck assignment problem, Manage. Sci. 14 (1968) 732-734. 
[24] D. Prabuddha, J. B. Ghosh, E. W. Charles, On the solution of a stochastic bottleneck assignment problem and its variations, Nav. Res. Log. 39 (1992) 389-397.

[25] U. Pferschy, The random linear bottleneck assignment problem, RAIROOper. Res. 30 (1996) 127-142.

[26] P. A. Krokhmal, P. M. Pardalos, Random assignment problems, Eur. J. Oper. Res. 194 (2009) 1-17.

[27] U. U. Haus, Bounding stochastic dependence, joint mixability of matrices, and multidimensional bottleneck assignment problems, Oper. Res. Lett. 43 (2015) 74-79.

[28] B. Liu, Uncertainty Theory: A Branch of Mathematics for Modeling Human Uncertainty, Springer-Verlag, Berlin, Germany, 2010.

[29] Y. H. Liu, Uncertain random programming with applications, Fuzzy Optim. Decis. Ma. 12 (2013) 153-169. 